\title{
A influência do nascimento de criança na atitude dos pais quanto à escolha do modo de transporte
}

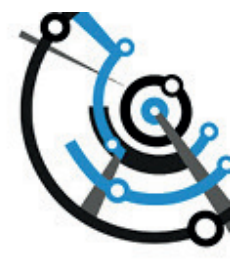

Fabiana Serra de Arruda

Departamento de Engenharia Civil e Ambiental e do Programa de Pós-Graduação em transportes da Universidade de Brasília, Brasil.

ORCID: https://orcid.org/oooo-00o2-5536-616o

\section{Marcelo Pereira Almeida}

Programa de Pós-Graduação em Transportes, Universidade de Brasília, Brasil

ORCID: https://orcid.org/oooo-ooo2-7353-6346

\author{
Daniel Pires Vieira \\ Programa de Pós-Graduação em Administração, Universidade de Brasília, Brasil. \\ ORCID: https://orcid.org/oooo-0002-2126-2750
}

Recibido: 13 de junio de 2020. Aceptado: 7 de marzo de 2021.

\begin{abstract}
Resumo
Este trabalho objetiva analisar a relação entre o nascimento do primeiro filho e a atitude dos pais quanto à escolha do modo de transporte utilizado. O nascimento do primeiro filho é apontado na literatura como evento de importância na decisão dos pais sobre qual modo de transporte utilizar. A pesquisa analisou a atitude dos pais em relação a cinco variáveis relativas aos deslocamentos, antes e após o nascimento do primeiro filho. Os dados usados foram coletados em domicílios com crianças até seis anos de idade. Os resultados mostram que quando não houve a mudança de modo de transporte após o nascimento da criança, não houve mudança de atitude dos pais. Esse resultado permite concluir que o nascimento da criança por si só não é condição suficiente para a mudança de atitude dos pais quanto ao modo de transporte usado.
\end{abstract}




\title{
The influence of childbirth on the parent's attitude regarding the transport mode choice
}

\begin{abstract}
This paper aims to analyze the relationship between the first child birth and parent's attitude regarding the transportation mode choice. First child birth is pointed out in the literature as an important event in parents' decision of which transportation mode to choose. This study analyzed the parents' attitude regarding five variables related to travel before and after the first child birth. The data used were collected in households with children up to six years old. The results show that when there was no change in transportation mode after the child's birth, there was no change in the parents' attitude. This result allows to conclude that the child's birth itself is not a sufficient condition for parent's attitude change related to transportation mode choice.
\end{abstract}

Keywords: Childbirth. Mobility. Transport mode. Attitude.

Palabras clave: Nacimiento de niños. Movilidad. Modo de transporte. Actitud.

\section{Introdução}

A tomada de decisão individual ou em um domicílio pela escolha do modo de transporte deve considerar a rotina diária daqueles indivíduos e as possíveis alterações que possam ocorrer nessa rotina (Schoend0uwe et al., 2015). A forma como as pessoas organizam sua agenda diária de atividades e como isso afeta suas escolhas de transporte vem sendo estudada por diversos pesquisadores, que apontam características como a idade, a renda, a localização da residência e do trabalho, o estilo de vida, os hábitos e as atitudes (Lanzendorf, 2003; Seraj et al.,2012; Davison e Ryley, 2013) como fatores significativos para a tomada de decisão. Assim, há um conjunto de características individuais e de composição domiciliar que acabam por influenciar na forma como essas pessoas decidem quanto à escolha do modo de transporte para seus deslocamentos.

Alguns autores explicam o comportamento individual de escolha do modo de transporte pela ocorrência de eventos específicos que ocorrem durante a vida, eventos esses que podem desencadear a necessidade de mudanças de comportamento relacionado à escolha da forma de deslocamento (van der Waerden et al., 2003; Lanzendorf, 2003; Scheiner, 2006; Oakil et al., 2011; Oakil, 2013). Esses eventos podem ser, por exemplo, o alcance da idade adulta, a aposentadoria ou o nascimento de um filho, e funcionam como gatilhos que causam significativas mudanças na forma como o indivíduo planeja e realiza seus deslocamentos (van der Waerden et al., 2003; Prillwitz et al., 2006; Scheiner, 2007; Lanzerdorf, 2010; Oakil, 2013; Schoenduwe et al., 2015).

McCarthy et al. (2017), Lanzendorf (2010) e Zwerts et al. (2010) identificam um conjunto de eventos que impactam na mudança de escolha do modo de transporte, dando destaque ao nascimento de uma criança. A presença de crianças em um domicílio, principalmente o primeiro filho, implica a reorganização ou flexibilização das agendas de atividades ora executadas. Os pais têm, então, a necessidade de rever suas decisões de forma a atender, além de suas próprias necessidades de deslocamento, aqueles referentes aos cuidados com criança (visitas a clínicas, escolas, passeios), uma vez que esses deslocamentos não podem ser realizados pela criança por si só. Para Lazendorf (2010) e Oakil (2013), o nascimento de criança é um dos eventos que mais afeta o comportamento de viagem em um domićlio.

Entretanto, de acordo com Zwerts et al. (2010) e McCarthy et al. (2017), ainda poucos estudos abordam a mudança de comportamento domiciliar após o nascimento da 
criança. Um estudo dessa natureza é importante para se entender os fatores que afetam a escolha modal nesse processo de transição e ajuste que acontece para os pais após o nascimento de seu primeiro filho. Tanto no brasileiro como em outros países da América do Sul, apesar do número considerável de estudos que envolvam fatores comportamentais relacionados aos deslocamentos individuais (por exemplo, Taco, 2003; Pitombo, 2007; Takano, 2010 e 2018), e aspectos relacionados à mobilidade urbanda (Alcalá e Scornik, 2015; Orellana et al., 2017; Jáuregui Díaz et al., 2020) não foram encontrados, até o presente momento, pesquisas que tenham com o foco específico o nascimento de crianças e sua influência no comportamento de viagens domiciliar.

Dado que o nascimento da criança altera a forma como os pais escolhem seu modo de transporte, entender as razões que os levam a essas mudanças é um importante passo para que se possa melhor pensar e planejar um sistema de transporte que atenda de forma adequada às necessidades dos indivíduos. A busca pelo entendimento de como determinadas condições influenciam o comportamento individual é o foco dos estudos que envolvem a Teoria do Comportamento Planejado (TCP) (Ajzen, 1991). De acordo com a teoria, o comportamento (ação em si) é pré-determinado pela intenção de uma pessoa em desempenhar determinado comportamento, que por sua vez é pré-determinada por um conjunto de componentes comportamentais, como a atitude. Entende-se atitude como sendo a avaliação ou percepção individual de que seu comportamento gera consequências favoráveis ou desfavoráveis, e que é um fator de influência sobre a escolha do modo de transporte (Ajzen, 1991; Bamberg et al., 2003; Anable, 2005; Gardner e Abraham, 2008).

Diante o exposto, o presente trabalho tem por objetivo analisar se o evento chave nascimento de criança afeta a atitude dos pais quanto ao modo de transporte usado e se esse evento por si só influencia a mudança de modo de transporte (o comportamento).

\section{Referencial teórico}

As relações entre comportamento e atitude já vem sendo estudada na área dos transportes em relação à escolha dos modos de transportes (Tertoolen et al. 1998; Nordfjaern et al., 2014; Simsekoglu et al. 2015). De acordo com a TCP, o comportamento é influenciado pela intenção de um indivíduo em desempenhar determinado comportamento. Assim, a escolha por um ou outro modo de transporte (comportamento) irá ocorrer a partir da influência de um conjunto de componentes comportamentais que fazem parte do indivíduo, dentre os quais se destaca a atitude como uma forte preditora do comportamento de escolha modal (Anable, 2005).

O estudo desenvolvido por Tertoolen et al. (1998) buscou verificar como o conhecimento dos danos ambientais e dos custos associados ao uso do automóvel influencia a atitude dos respondentes quanto ao uso desse modo. O estudo identificou, inicialmente, que aspectos como conforto, independência, custo, ambiente e segurança eram considerados como vantajosos pelos respondentes sobre o uso do automóvel. Após os entrevistados serem expostos aos aspectos ambientais e econômicos negativos do uso do carro, verificou-se que eles apresentaram uma tendência de mudança de atitude em relação ao uso do automóvel (maior consciência ambiental), mas não de mudança de comportamento em si. Ou seja, a consciência de que o uso desse modo traz prejuízos ambientais coletivos, não direcionou a uma mudança de comportamento. Os autores argumentam que o uso do automóvel está associado ao sentimento que as pessoas têm de maior independência e conveniência pelo uso do carro.

O estudo de Nordfjaern et al. (2014) avaliou como os componentes da TCP (atitude, normas subjetivas e comportamento percebido), o hábito do uso do automóvel e a resistência 
em mudar afetavam o uso do transporte público. Os resultados encontrados mostraram que atitudes favoráveis ao uso do transporte coletivo, quando avaliadas juntamente com o hábito e a resistência em mudar, tinham uma influência fraca sobre a intenção de uso do transporte público. O estudo revela que, dentre os componentes da TCP, as normas subjetivas, aquelas construídas pelo indivíduo com base em suas crenças pessoais, estão fortemente associadas à intenção de uso do transporte público. Isso significa dizer que o papel social tem importância na mudança de escolha modal podendo o indivíduo ser influenciado pelo comportamento de outros indivíduos, o que tem papel importante na escolha modal de crianças, que tendem a acompanhar as escolhas modais de seus pais.

Şimşekoğlu et al. (2015) mediram a atitude dos indivíduos frente ao uso do transporte público a partir de afirmações avaliadas em uma escala de sete pontos, variando de concordo totalmente a discordo totalmente. Os resultados mostraram que os usuários do transporte público e promotores do transporte saudável, apresentaram atitude favorável quanto ao uso desse modo, enquanto o hábito de uso do automóvel foi um preditor negativo do uso do transporte público. Os usuários do automóvel apresentaram uma associação negativa ao uso do transporte público devido a barreiras percebidas, como dificuldades de viajar com crianças e nível social. Ao contrário do estudo do Tertoolen et al. (1998), a atitude aparece como um preditor significativo no uso do transporte público.

O estudo da ocorrência de eventos-chave como influenciadores do comportamento de escolha do modo de transporte é outra relação também abordada por diversos pesquisadores (van der Waerden et al., 2003; Oakil et al., 2011; Scheiner, 2014; McCarthy, 2017). Os estudos indicam que eventos que ocorrem ao longo da vida de uma pessoa podem ser significativos em suas escolhas de modo de transporte ou localização residencial, mudando seu hábito e seu comportamento (Axhausen et al., 2001; Lanzendorf, 2003; Oakil, 2013). Ou seja, qualquer alteração que ocorra na vida do indivíduo, que funcione como um marco em sua vida, leva a uma alteração na forma como as escolhas do modo de transporte ocorrem. Seja devido ao alcance da vida adulta, ao primeiro emprego remunerado ou à mudança de emprego, o indivíduo poderá, baseado em suas preferências e necessidades, alterar suas escolhas. Essas alterações irão refletir, de certa forma, as preferências por um ou outro modo, a partir das características desses modos (como maior flexibilidade de trajeto ou menor tempo de viagem) e as necessidades atuais de cada indivíduo (por exemplo mobilidade reduzida e levar crianças ou maior conforto).

Klöckner (2004) identificou os eventos mais importantes que ocorrem na vida de um indivíduo e os resultados indicaram que a obtenção da carteira de habilitação é aquela que mais afeta a escolha do modo de transporte. Reforça essa ideia a análise de Scheiner (2007), ao expressar que a estrutura doméstica desempenha um papel importante para a motorização das famílias, inclusive ao afirmar que a motorização em um domicílio muda consideravelmente com o aumento ou a redução no número de familiares. Para o autor, com a chegada do primeiro filho, a dependência do carro fica mais intensa para as mães, e com o segundo, torna-se indispensável.

Consequentemente, as decisões para os deslocamentos geram impacto no comportamento de viagem, em especial no modo de alterar as decisões de escolhas (Lanzendorf et al., 2015). Dessa forma, ao analisar os eventos e os seus impactos sobre os padrões diários de atividade e de viagem, há uma significativa contribuição para a mudança no foco da análise baseada em atividade e nos padrões de viagem ao longo de vários horizontes temporais (Oakil, 2013).

Para Bernardo et al. (2015) os estudos de comportamento de viagem que consideram a estrutura domiciliar, contribuem para o redesenho das políticas governamentais e 
facilitam na redução do conflito existente entre o trabalho e a família. Além disso, alguns questionamentos referentes aos estudos comportamentais para o deslocamento, ao longo do tempo, precisam ser melhor explorados quando se tratam da ocorrência de eventos-chave. Conforme exposto por Neri et al. (2020), o uso de pesquisas domiciliares, que contenham informações referentes à escolha de modo de transporte para centros educacionais infantis, são ferramentas úteis para o processo de formulação de políticas públicas voltadas à melhoria do transporte e da mobilidade em meios urbanos.

Ao estudar as mudanças nos padrões de atividades e viagem ao longo do tempo, Scheiner (2014) evidenciou uma nítida diferença na análise dos eventos por gênero, chegando a sugerir que os homens e as mulheres são diferentemente afetados por eventos ao longo do curso de vida. $\mathrm{O}$ pesquisador verificou que eventos-chave como o nascimento de criança, a entrada no mercado de trabalho, as mudanças no contexto espacial, a acessibilidade e a mobilidade apresentam significativa diferença por gênero. Os resultados mostraram que as viagens realizadas pelas mulheres são mais complexas e aumentam mais que as dos homens ao longo do tempo. Existem poucos eventos que exibem a distinção específica de gênero. Os filhos, ao saírem da casa dos pais, ocasionam a diminuição dos padrões de atividades, isso se deve ao compartilhamento familiar das atividades. O número e a idade dos filhos são considerados fatores de impacto na repartição do trabalho familiar. As mães têm maiores obrigações familiares e estas responsabilidades aumentam com o número de filhos e, gradativamente, diminuem com a idade do filho mais novo (Scheiner, 2014).

O evento nascimento de criança é considerado como um dos marcos que afetam a escolha do modo de transporte. Geralmente, esse evento está ligado à escolha do automóvel para a realização de viagens, com os argumentos de terem que carregar muitos acessórios, precisar ter flexibilidade no horário, necessidade de encadeamento de viagens. Segundo Zwets et al. (2010) e McCarthy et al. (2017), a maior parte dos estudos desenvolvidos para identificar fatores que influenciam a escolha modal de famílias com crianças, tendem a indicar o uso do automóvel, seja por praticidade ou conforto.

Scheiner e Holz-Rau (2013) ao estudar as alterações quanto ao modo de viagem de um ano para o outro a partir de uma base de dados de três anos consecutivos, verificou que o nascimento de criança apresenta uma maior representatividade para as mães. Os modos mais utilizados são o automóvel (as mães sendo motoristas) e a realização de caminhadas. As mudanças ocorrem principalmente para as mães pois, para os pais, $o$ nascimento de criança não parece ter um efeito sobre o uso dos modos de transporte.

Apesar dos estudos afirmarem que há alteração de modo de transporte após o nascimento de um filho, Lanzendorf (2010) concluiu que a troca para o automóvel pode não ter causa apenas no nascimento de criança. No estudo realizado, o autor observou um contraste à crença comum que o nascimento de crianças favorece a aquisição do carro e à frequência de uso do automóvel. Verificou-se que mesmo após o nascimento da criança, as mães não sentiram a necessidade de adquirir a carteira de habilitação, optando por realizar os deslocamentos por outros modos alternativos ao automóvel.

\section{Método}

A presente pesquisa se caracteriza como descritiva exploratória, com a aplicação de métodos quantitativos. As análises foram realizadas a partir de informações primárias coletadas por meio de questionário eletrônico direcionado ao responsável pelos deslocamentos da criança e seu cônjuge. A pesquisa foi direcionada aos respondentes que satisfaziam a condição de ter tido o primeiro filho há pelo menos 6 anos. Entende-se 
que o período de 6 anos é adequado para que os pais se recordem do efeito ocorrido a partir do nascimento do primeiro filho. Outra recomendação foi que as mães ou pais já tenham passado pelo período de licença maternidade/ paternidade, pois estes são momentos atípicos em que os pais e mães não realizam os deslocamentos habituais.

Foi utilizada a abordagem retrospectiva pois, com base na literatura, existem datas significativas na vida do indivíduo, marcadas por eventos específicos (por exemplo, formatura, casamento, nascimento de filhos, mudança no trabalho) e que acontecem com baixa frequência e são fáceis de serem lembradas com razoável precisão (van der Waerden et al., 2003; Lanzendorf, 2003 e 2010; Lanzendorf et al. 2015).

O questionário era composto por perguntas divididas em 3 blocos, conforme pode ser encontrado em Almeida (2016). O primeiro bloco buscava coletar informações sociodemográficas sobre o respondente e seu cônjuge. Posteriormente eram feitas perguntas sobre o principal meio de transporte utilizado e a sua atitude quanto ao grau de importância atribuído aos aspectos referentes à escolha daquele modo antes do nascimento do primeiro filho. Por fim, eram realizadas perguntas sobre o meio de transporte utilizado e a sua atitude quanto ao grau de importância atribuído a determinados aspectos referentes à escolha daquele modo atualmente, após o nascimento do primeiro filho. Foram avaliadas as variáveis segurança, tempo de deslocamento, conforto/conveniência, custo, flexibilidade no deslocamento, qualidade do transporte público e questões ambientais a partir de uma escala de 5 pontos, variando de "sem importância" (1) a "muito importante" (5). Ressalta-se que os entrevistados não foram instruídos quanto aos conceitos das variáveis analisadas, sendo as respostas a representação do entendimento de cada respondente quanto aos aspectos citados.

A coleta de dados aconteceu entre os meses de fevereiro e março de 2016, no Distrito Federal, Brasil, e foram obtidos 234 questionários considerados válidos. Ressalta-se que apenas os questionários dos respondentes que atendiam ao critério do primeiro filho ter nascido há pelo menos seis anos foram validados. Os dados foram analisados inicialmente com estatística descritiva para compreensão das características da amostra obtida. Como forma de verificar a mudança na percepção dos indivíduos quanto a relevância das características do modo de transporte selecionado, foram realizados testes de comparação de médias. De forma específica, foi empregado o Teste $t$ para amostras dependentes (ou amostras emparelhadas). Esse tipo de teste é usado quando para um mesmo grupo amostral existem duas condições experimentais analisadas (Field, 2009). Para fins do presente estudo, foram consideradas como condições a percepção anterior e posterior ao nascimento do primeiro filho. Na seção seguinte são apresentados os resultados das análises estatísticas.

\section{Resultados e análises}

O questionário foi aplicado ao respondente responsável pelos deslocamentos da criança e cônjuge/companheiro, caso existisse. Nesse tocante, $69.66 \%$ dos(as) respondentes informaram ser parceiro(a) ou cônjuge. Por outro lado, $26.50 \%$ assumiram o papel de chefe de família e $3.85 \%$ de filho(a), isto é, indivíduos que, apesar de terem filhos, ainda residem com os pais.

A amostra pesquisada é composta em sua maioria por mulheres $(83,8 \%)$. Em relação ao grau de instrução, a amostra é composta em sua maioria por pessoas com graus de instrução elevados (31\% com ensino superior completo e $51,7 \%$ com pós-graduação). Com relação a ocupação, mais de $80 \%$ dos respondentes possui alguma ocupação formal. Nota-se uma concentração dos respondentes nos extratos intermediários e 
superiores de renda, sendo que mais de $60 \%$ da amostra possui renda familiar acima de 4 salários mínimos, o equivalente a US $\$ 975.00$ (valores de março de 2016). Em relação à idade, verificou-se uma predominância de respondentes com idades entre 29 e 40 anos.

Ainda considerando as informações para caracterização dos respondentes, observa-se que houve um aumento no número de carteiras de habilitação após o nascimento da criança, principalmente para as mulheres $(11,11 \%)$. Cerca de $15 \%$ dos domicílios não possuía automóvel antes do nascimento do filho e os demais possuíam, no mínimo, um automóvel. Após o nascimento da criança, observou-se uma alteração referente à aquisição do automóvel, sendo que o percentual de domicílios sem automóvel reduziu de $15 \%$ para $5,98 \%$, indicando que a chegada da criança pode ter induzido à compra do automóvel, como apontam os estudos de Scheiner (2006).

Para 88,03\% da amostra geral, considerando homens e mulheres, o número de atividades e deslocamentos realizados semanalmente aumentou após o nascimento da criança. Para o segmento feminino, $89,28 \%$ das respondentes afirmaram que suas atividades aumentaram durante a semana, o que corrobora com estudos realizados por Zwerts et al. (2010). Essa variação das atividades é esperada, visto que além dos deslocamentos usuais realizados, a criança demanda outros atendimentos, como visitas ao médico e escola por exemplo.

Considerando o modo de transporte utilizado antes e após o nascimento do primeiro filho, pode-se observar -a partir da Figura 1- que há um incremento do uso do automóvel (seja dirigindo - 14\%, seja por carona - 1,7\%) após o nascimento da criança. O crescimento observado é compensado por um declínio do uso de ônibus, motos e da caminhada como modo de transporte. Assim, percebe-se que o aumento no número de viagens por automóvel decorre da migração de usuários dos outros modos de transporte. Em que pese essas diferenças observadas, cumpre destacar que para $65 \%$ da amostra pesquisada não houve mudança no meio de transporte utilizado após o nascimento do primeiro filho.

As primeiras análises realizadas buscaram evidenciar os efeitos do nascimento do primeiro filho na importância das características atribuídas ao modo de transporte utilizado. Para análise foi empregado o Teste $t$ para amostras emparelhadas. Foram comparadas as respostas quanto às variáveis citadas no item 3 deste trabalho, para o período anterior e posterior ao nascimento do primeiro filho. Os resultados são apresentados no Quadro 1.

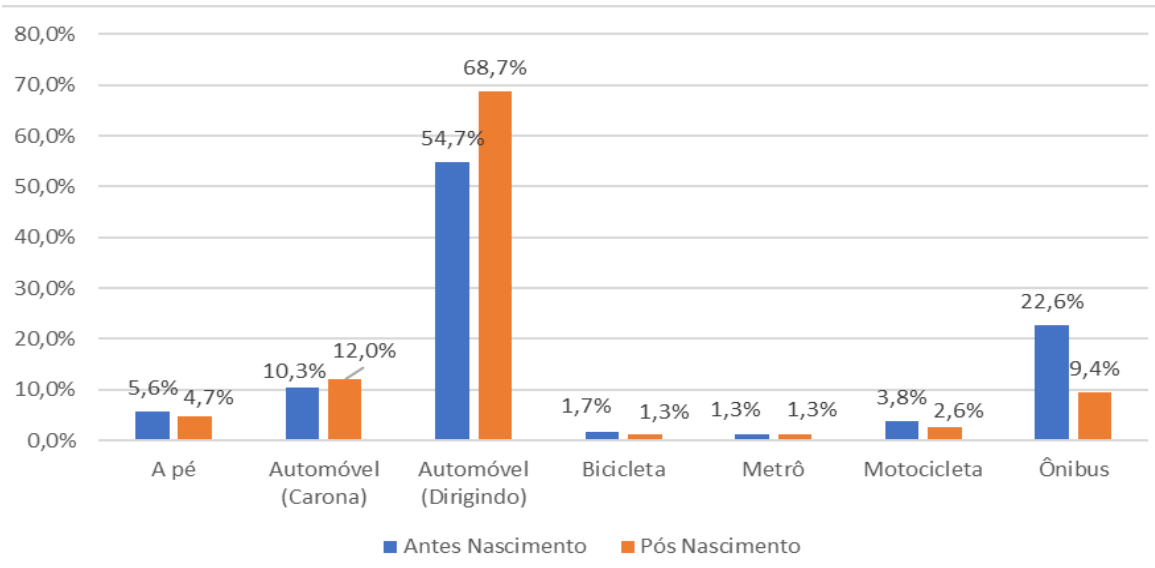

Figura 1. Comparativo dos Modos de Transporte. Fonte: elaboração própria com dados da pesquisa. 
Quadro 1. Comparação Médias -Antes e Pós Nascimento-. Fonte: elaboração própria com dados da pesquisa.

\begin{tabular}{|c|c|c|c|c|c|}
\hline Variáveis & & Média & $\begin{array}{l}\text { Dif. Médias } \\
\text { (Pós - Antes) }\end{array}$ & $t$ & p-value. \\
\hline \multirow{2}{*}{ Segurança } & Pós nasc. & 4,31 & \multirow{2}{*}{0,25} & \multirow[b]{2}{*}{4,394} & \multirow{2}{*}{0,000} \\
\hline & Antes nasc. & 4,06 & & & \\
\hline \multirow{2}{*}{$\begin{array}{l}\text { Tempo de } \\
\text { deslocamento }\end{array}$} & Pós nasc. & 4,37 & \multirow{2}{*}{0,21} & \multirow{2}{*}{3,61} & \multirow{2}{*}{0,000} \\
\hline & Antes nasc. & 4,16 & & & \\
\hline \multirow{2}{*}{$\begin{array}{l}\text { Conforto/ } \\
\text { Conveniência }\end{array}$} & Pós nasc. & 4,24 & \multirow{2}{*}{0,22} & \multirow{2}{*}{3,619} & \multirow{2}{*}{0,000} \\
\hline & Antes nasc. & 4,02 & & & \\
\hline \multirow{2}{*}{ Custo } & Pós nasc. & 3,88 & \multirow{2}{*}{0,02} & \multirow{2}{*}{0,349} & \multirow{2}{*}{0,728} \\
\hline & Antes nasc. & 3,86 & & & \\
\hline \multirow{2}{*}{ Flexibilidade } & Pós nasc. & 4,29 & \multirow[b]{2}{*}{0,19} & \multirow[b]{2}{*}{3,332} & \multirow[b]{2}{*}{0,001} \\
\hline & Antes nasc. & 4,10 & & & \\
\hline \multirow{2}{*}{$\begin{array}{l}\text { Qualidade do } \\
\text { transporte público }\end{array}$} & Pós nasc. & 4,01 & \multirow[b]{2}{*}{0,19} & \multirow[b]{2}{*}{2,704} & \multirow[b]{2}{*}{0,007} \\
\hline & Antes nasc. & 3,82 & & & \\
\hline \multirow{2}{*}{ Ambiente } & Pós nasc. & 3,81 & \multirow{2}{*}{0,10} & \multirow{2}{*}{1,707} & \multirow{2}{*}{0,089} \\
\hline & Antes nasc. & 3,71 & & & \\
\hline
\end{tabular}

Os resultados encontrados indicam que há mudanças significativas ( $p$-value $<0,05)$ na atitude dos respondentes após o nascimento da criança para as variáveis: segurança, tempo de deslocamento, conforto/conveniência, flexibilidade e qualidade do transporte público. Nesses casos, verifica-se que a amplitude na diferença entre as médias na percepção dos entrevistados, é suficiente para dar significância à variável. Por outro lado, observa-se que para as variáveis custo e ambiente, os resultados estatísticos foram não significativos, sendo a amplitude da diferença entre as médias pequena. Esse resultado indica que a percepção dos entrevistados não mudou após o nascimento da criança. Ressalta-se que a diferença entre as médias das variáveis é positiva, indicando que há um aumento na percepção da relevância dessas variáveis após o nascimento da criança. É interessante notar que, após o nascimento da criança, a atitude sobre a qualidade do transporte público aumentou (média de 3,82 para 4,01 ) mas o percentual de pessoas usando esse modo para seus deslocamentos caiu de $22,6 \%$ para $9,4 \%$ após o nascimento da criança (Figura 1). Isso pode ser explicado pelas outras variáveis significativas como segurança e tempo de deslocamento.

Conforme exposto, após o nascimento da criança, houve aumento no número de atividades e deslocamentos realizados pelos responsáveis. Assim, apesar da atitude quanto a qualidade do transporte coletivo ter aumentado, não houve migração para esse modo, já que eventualmente não ofereça os níveis desejados de segurança e flexibilidade para a realização das atividades dentro de um determinado período de tempo. Aspectos como o conforto e o tempo de viagem também são relevantes quando se aborda o deslocamento com crianças (McCarthy et al., 2017).

A ausência de diferença para a variável custo pode ser explicada pela homogeneidade da renda na amostra pesquisada. A maior parte dos entrevistados pertencem a uma mesma categoria de renda, o que pode indicar que o custo do transporte não tem efeito significativo na escolha de um ou outro modo de transporte. A variável Ambiente, que buscou medir a percepção dos respondentes quanto aos danos trazidos pelo modo de transporte usado ao meio ambiente, como ruído e poluição atmosférica, não apresentou valores significativos dentro do parâmetro estabelecido. Os resultados 
dessas duas variáveis são contrários àqueles encontrados por Tertoolen et al. (1998), uma vez que a atitude em relação às variáveis medidas não sofreu alterações após o nascimento da criança.

Considerando que a atitude quanto à mudança de modo de transporte após o nascimento do primeiro filho é aspecto central deste estudo, optou-se por dividir a amostra em dois subgrupos: (i) grupo em que não houve mudança de modo de transporte (65\% da amostra original); e (ii) grupo em que houve mudança no modo de transporte ( $35 \%$ da amostra original). A partir dessa subdivisão, foram realizadas novas comparações a partir do Teste $t$ pareado comparando as percepções anterior e posterior ao nascimento dentro dos subgrupos. O Quadro 2 apresenta os resultados do Teste $t$ pareado para o subgrupo em que houve mudança do modo de transporte.

Quadro 2. Comparação Médias -Antes e Pós Nascimento-. Subgrupo com Mudança de Modo de Transporte. Fonte: elaboração própria com dados da pesquisa.

\begin{tabular}{|c|c|c|c|c|c|}
\hline Variáveis & & Média & $\begin{array}{l}\text { Dif. Médias } \\
\text { (Pós - Antes) }\end{array}$ & $t$ & p-value. \\
\hline \multirow{2}{*}{ Segurança } & Pós nasc. & 4,26 & \multirow{2}{*}{0,53} & \multirow{2}{*}{4,507} & \multirow{2}{*}{0,000} \\
\hline & Antes nasc. & 3,73 & & & \\
\hline \multirow{2}{*}{ Tempo } & Pós nasc. & 4,28 & \multirow{2}{*}{0,55} & \multirow{2}{*}{4,464} & \multirow[b]{2}{*}{0,000} \\
\hline & Antes nasc. & 3,73 & & & \\
\hline \multirow{2}{*}{$\begin{array}{l}\text { Conforto/ } \\
\text { Conveniência }\end{array}$} & Pós nasc. & 4,13 & \multirow{2}{*}{0,52} & \multirow{2}{*}{3,751} & \multirow{2}{*}{0,000} \\
\hline & Antes nasc. & 3,61 & & & \\
\hline \multirow{2}{*}{ Custo } & Pós nasc. & 3,94 & \multirow{2}{*}{0,12} & \multirow{2}{*}{0,886} & \multirow{2}{*}{0,379} \\
\hline & Antes nasc. & 3,82 & & & \\
\hline \multirow{2}{*}{ Flexibilidade } & Pós nasc. & 4,27 & \multirow{2}{*}{0,46} & \multirow{2}{*}{4,114} & \multirow{2}{*}{0,000} \\
\hline & Antes nasc. & 3,81 & & & \\
\hline \multirow{2}{*}{ Qualidade } & Pós nasc. & 3,99 & \multirow{2}{*}{0,36} & \multirow{2}{*}{2,518} & \multirow{2}{*}{0,014} \\
\hline & Antes nasc. & 3,63 & & & \\
\hline \multirow{2}{*}{ Ambiente } & Pós nasc. & 3,72 & \multirow{2}{*}{0,35} & \multirow{2}{*}{2,838} & \multirow{2}{*}{0,006} \\
\hline & Antes nasc. & 3,37 & & & \\
\hline
\end{tabular}

Os resultados encontrados indicam a ocorrência de diferenças significativas ( $p$-value $<0,05)$ antes e pós nascimento para as variáveis: segurança, tempo, conforto/conveniência, flexibilidade, qualidade do transporte público e ambiente. Observar-se que a amplitude na diferença entre as médias na percepção dos entrevistados, é suficiente para dar significância à variável. Vale observar que as amplitudes das médias para este grupo são maiores que aquelas mostradas no Quadro 1, o que pode ser um indicativo de maior atribuição de valor a essas variáveis quando há mudança de modo de transporte. Todas as diferenças entre as médias são positivas, indicando um aumento da percepção. A exemplo da comparação para a totalidade da amostra não há diferença significativa para a variável Custo, o que pode ser justificado pela alta renda dos respondentes. Nota-se que a variável Ambiente, diferentemente do encontrado na análise anterior, apresentou resultados significativos, o que indica uma mudança de atitude (maior consciência ambiental). Da mesma forma que a análise anterior, todas as demais variáveis foram significativas e positivas.

Tal como nas análises anteriores, procedeu-se a comparação da atitude antes e depois do nascimento para o subgrupo em que não houve mudança no meio de transporte. O Quadro 3 apresenta os resultados encontrados. 
Quadro 3. Comparação Médias -Antes e Pós Nascimento-. Subgrupo sem Mudança de Modo de Transporte. Fonte: elaboração própria com dados da pesquisa.

\begin{tabular}{|c|c|c|c|c|c|}
\hline Variáveis & & Média & $\begin{array}{l}\text { Dif. Médias } \\
\text { (Pós - Antes) }\end{array}$ & $t$ & $\mathrm{p}$-value. \\
\hline \multirow{2}{*}{ Segurança } & Pós nasc. & 4,33 & \multirow{2}{*}{0,1} & \multirow{2}{*}{1,744} & \multirow{2}{*}{0,083} \\
\hline & Antes nasc. & 4,23 & & & \\
\hline \multirow{2}{*}{ Tempo } & Pós nasc. & 4,42 & \multirow{2}{*}{0,03} & \multirow{2}{*}{0,592} & \multirow{2}{*}{0,555} \\
\hline & Antes nasc. & 4,39 & & & \\
\hline \multirow{2}{*}{$\begin{array}{l}\text { Conforto/ } \\
\text { Conveniência }\end{array}$} & Pós nasc. & 4,3 & \multirow{2}{*}{0,06} & \multirow{2}{*}{1,197} & \multirow{2}{*}{0,233} \\
\hline & Antes nasc. & 4,24 & & & \\
\hline \multirow{2}{*}{ Custo } & Pós nasc. & 3,86 & \multirow{2}{*}{$-0,02$} & \multirow{2}{*}{$-0,395$} & \multirow{2}{*}{0,693} \\
\hline & Antes nasc. & 3,88 & & & \\
\hline \multirow{2}{*}{ Flexibilidade } & Pós nasc. & 4,3 & \multirow{2}{*}{0,06} & \multirow{2}{*}{0,842} & \multirow{2}{*}{0,401} \\
\hline & Antes nasc. & 4,24 & & & \\
\hline \multirow{2}{*}{ Qualidade } & Pós nasc. & 4,03 & \multirow{2}{*}{0,11} & \multirow{2}{*}{1,356} & \multirow{2}{*}{0,177} \\
\hline & Antes nasc. & 3,92 & & & \\
\hline \multirow{2}{*}{ Ambiente } & Pós nasc. & 3,86 & \multirow{2}{*}{$-0,03$} & \multirow{2}{*}{$-0,569$} & \multirow{2}{*}{0,571} \\
\hline & Antes nasc. & 3,89 & & & \\
\hline
\end{tabular}

Ao analisar a subamostra dos casos em que não houve mudança no meio de transporte, os resultados indicam que não houve mudança significativa na atitude dos respondentes para as variáveis pesquisadas, observando-se a pequena margem de amplitude entre as médias. Os resultados mostram que para o grupo em que não houve a mudança de modo de transporte a atitude quanto aspectos como tempo de deslocamento, conforto e flexibilidade permanece a mesma apesar do nascimento da criança. A exceção pode ser encontrada para a variável Segurança que apresentou diferença significativa ( $\mathrm{p}$-value $<0,1$ ), indicando que o nascimento do primeiro filho acarreta maior atenção nesse aspecto de seleção do modo de transporte, mesmo quando não há mudança no meio de transporte utilizado.

Os resultados das análises das subamostras (com e sem mudança de meio de transporte) permitem inferir que o nascimento da criança por si só não é uma condição suficiente para a mudança de atitude (uma vez que quando não há mudança do meio de transporte, não há mudança da atitude). Esses resultados vão ao encontro daqueles encontrados por Lanzerdorf (2010). Apesar dos métodos de análise diferenciados aplicados nos trabalhos, os dois resultados mostram que as mudanças de modos de transporte após o nascimento de uma criança podem estar sendo afetadas por outras questões. Os resultados encontrados permitem argumentar que o nascimento da criança por si só não está associado a mudança de atitude quanto às características do meio de transporte. Essas mudanças somente foram observadas quando acompanhadas de alterações no meio de transporte utilizado.

\section{Conclusão}

De acordo com a literatura, o comportamento individual quanto à escolha do modo de transporte pode variar em função de eventos que ocorrem em sua vida. A mudança no comportamento de escolha do modo está relacionada a estágios do ciclo de vida do indivíduo, em que determinados eventos podem afetar diretamente a mudança do modo de transporte. A mudança do modo de transporte pode, ainda, ter relação com as percepções dos indivíduos quanto ao modo de transporte. Essa percepção varia em função das características tanto do indivíduo quanto de seu domicílio. 
Neste trabalho, explorou-se como o nascimento do primeiro filho afeta a atitude dos indivíduos quanto ao modo de transporte usado. A hipótese adotada é que o filho influencia na atitude dos pais quanto à escolha de um modo de transporte, levando a uma mudança de comportamento. Os resultados que contemplam o total da amostra pesquisada mostram que $o$ as relações comumente encontradas na literatura, que relacionam o evento crítico nascimento de criança com a atitude e comportamento sobre a escolha do modo de transporte, se confirma neste estudo.

Tomando como base os resultados obtidos a partir da análise dos dois subgrupos, observa-se que a mudança de atitude dos indivíduos quanto aos atributos do modo de transporte usado, deve ser acompanhada por mudança do meio de transporte utilizado. Se não há mudança do modo de transporte, não há mudança de atitude, apesar do nascimento da criança. Nesse aspecto, o que gera a mudança do modo de transporte podem estar mais relacionados a fatores como hábito ou preferências pessoais, ou outros componentes da TCP, do que a ocorrência de eventos críticos. Esses resultados permitem questionar se o que efetivamente ocasiona a mudança de percepção não é a mudança de meio de transporte.

Diante do exposto, conclui-se que o nascimento da criança por si só não é condição suficiente para a mudança de atitude quanto ao modo de transporte usado. A mudança de atitude só ocorre quando ocorre a mudança do modo, independente do nascimento da criança. Assim, verifica-se a necessidade de medidas que visem mudar a percepção dos indivíduos quanto aos modos de transportes e suas características, de forma a que essa mudança de percepção seja suficiente para mudar a atitude em relação ao comportamento. Ou seja, ao mudar a forma como a pessoa compreende os pontos positivos e negativos de sua escolha, ela pode ter seu comportamento alterado.

Dentre as limitações do trabalho, destaca-se a distribuição da amostra, que é residente do Distrito Federal e tem, em sua maioria, curso superior e renda acima de quatro salários mínimos. A amostra foi definida por conveniência, uma vez que há a dificuldade em se encontrar pessoas disponíveis para responder que atendessem ao critério do nascimento do primeiro filho ter sido até 6 anos. $O$ fato da renda dos respondentes estar concentrada em faixas mais altas, pode ser um indicativo de que o custo não é relevante para esse estrato pesquisado.

Outra limitação é que o avaliou apenas um dos componentes da TCP -a atitude. É importante que componentes como as normas subjetivas e o comportamento percebido também sejam avaliados, de forma a se obter um panorama mais claro sobre as razões pelas quais os indivíduos alteram seu comportamento de escolha modal frente a eventos que acontecem em sua vida.

Por se tratar de um exploratório, futuras análises podem ser feitas com base em amostras maiores, distribuídas em outras localidades e mais equilibrada em termos de faixas de renda. Outro aspecto que pode ser inserido nas análises é a localização espacial das atividades que são realizadas, uma vez que as condições de acessibilidade e mobilidade podem influenciar na decisão por um ou outro modo de transporte. 


\section{Q Bibliografia}

" Ajzen, I. (1991). The Theory of Planned Behavior. Organizational Behavior and Human Decision Processes, 50, p. 179-211.

"Alcalá, L. e Scornik, M. (2015). Movilidad y accesibilidad en el Gran Resistencia. Principales problemas y desafíos. Revista Transporte Y Territorio, 13, p. 8-35. https://doi.org/10.34096/rtt.i13.1875

» Almeida, M. P. (2016). A influência do evento-chave Nascimento de crianças no comportamento de viagem individual a partir da teoria "Biografias de Mobilidade". Dissertação de Mestrado. Programa de Pós-Graduação em Transportes, Universidade de Brasília.

»Anable, J. (2005). Complacent Car Addicts or Aspiring Environmentalists? Identifying Travel Behaviour Segments Using Attitude Theory. Transport Policy, V.12 (1), p. 65-78.

"Axhausen, K.W.; Zimmermann A.; Schönfelder S.; Rindsfüser G. e Haupt T. (2001). Observing the Rhythms of Daily Life: A six-week travel diary. Transportation, 29 (2), p. 95-124.

" Bamberg, S.; Rölle, D. e Weber, C. (2003). Does Habitual Car Use not Lead to More Resistance to Change of Travel Mode? Transportation, 30, p. 97-108.

» Bernardo, C.; Paleti, R.; Hoklas, M. e Bhat, C. (2015). An Empirical Investigation Into the Time-Use and Activity Patterns of Dual-Earner Couples With and Without Young Children. Transportation Research Part A, 69, p. 71-91.

»Davison, L. e Ryley, T. (2013). The Relationship Between Air Travel Behaviour and The Key Life Stages of Having Children and Entering Retirement. Journal of Transport Geography, 26, p. 78-86.

» Field, A. (2009). Descobrindo a Estatística usando o SPSS. $2^{2}$ Edição, Porto Alegre: Artmed.

" Gardner, B. e Abraham, C. (2008). Psychological Correlates of Car Use: A MetaSnalysis. Transportation Research Part F, 11, p. 300-311.

» Jáuregui Díaz, J. A.; Ávila Sánchez, M. de J. e Tovar Cabañas, R. (2020). Movilidad cotidiana de la población trabajadora en la Zona Metropolitana de Monterrey, 2015. Revista Transporte Y Territorio, 23, p. 201-221. https://doi.org/10.34096/rtt.i23.9663

» Klöckner, C. (2004). How Single Events Change Travel Mode Choice - a life span perspective. 4th International Conference of Traffic and Transport Psychology, Nottingham, 5-9. September 2004.

» Lanzendorf, M.; Shoenduwe, R.; Mueller, M. G. e Peters, A. (2015). Analysing Mobility Biographies With the Life Course Calendar: a Retrospective Survey Methodology for Longitudinal Data Collection. Jornal of Transport Geography, 42, p. 98-109.

»Lanzerdorf, M. (2010). Key Events and Their Effect on Mobility Biographies: the Case of Childbirth. International Journal of Sustainable Transportation, 4 (5), p. 272-292. https://doi.org/10.1080/15568310903145188

" Lazendorf, M.(2003). Mobility biographies. ANew Perspective for Understanding Travel Behaviour. 10th International Conference on Travel Behaviour Research. Utrecht University, Urban Research Centre. Disponível em: http://www.ivt. ethz.ch/news/archive/20030810_IATBR/lanzendorf.pdf(24/02/2015). 
» McCarthy, L.; Delbosc, A.; Currie, G. e Molloy, A. (2017). Factors Influencing Travel Mode Choice Among Families with Young Children (aged 0-4): a review of the literature. Transport Reviews, 37 (6), p. 767-781. https://doi.or g/10.1080/01441647.2017.1354942

» Neri, N. V.; Galeota, C. e Capelli, L. (2020). La utilización de las encuestas de movilidad domiciliaria para un análisis de accesibilidad: Aplicación para el caso de acceso a Centros Educativos en el Área Metropolitana de Buenos Aires. Revista Transporte y Territorio, 23, p. 71-81. https://doi.org/10.34096/rtt.i23.9657

» Nordfjaern, T.; Simsekoglu, Ö. e Rundmo, T. (2014). The Role of Deliberate Planning, Car Habit and Resistance to Change in Public Transportation Mode Use. Transport Research Part F, 27, p. 90-98.

»Oakil, A. T. M. (2013). Temporal Dependence in Life Trajectories and Mobility Decisions. Thesis of Faculty of Geosciences, Utrecht University, The Netherlands.

» Oakil, A. T. M.; Ettema, D.; Arentze, T. e Timmermans, H. (2011). A Longitudinal Analysis of the Dependence of the Commute Mode Switching Decision on Mobility Decisions and Life Cycle Events. Proceedings of the 16th International Conference of Hong Kong Society for Transportation Studies. Hong Kong, China.

»Orellana, D.; Hermida, C. e Osorio, P. (1). Comprendiendo los patrones de movilidad de ciclistas y peatones. Una síntesis de literatura. Revista Transporte Y Territorio, 16, p. 167-183. https://doi.org/10.34096/rtt.i16.3608

"Pitombo, C.S. (2007). Estudos de Relações Entre Variáveis Socioeconômicas, de Uso do Solo, Participação em Atividades e Padrões de Viagem Encadeadas Urbanas. Tese de Doutorado. Programa de Pós-Graduação em Engenharia de Transportes, Escola de Engenharia de São Carlos, Universidade de São Paulo. São Carlos, SP.

»Prillwitz, J.; Harms, S. e Lanzendorf, M., (2006). Impact of Life-Course Events on Car Ownership. Journal of The Transportation Research Record, 1985 (1), p. 71-77.

» Scheiner, J. (2006). Housing Mobility and Travel Behaviour: a Process-Oriented Approach to Spatial Mobility. Evidence from a new research field in Germany. Journal of Transport Geography, 14 (4), p. 287-298.

»Scheiner, J. (2007). Mobility Biographies Elements of a Biographical Theory of Travel Demand. Erdkunde 61 (2), p. 161-173. Disponível em: https://www. erdkunde.uni-bonn.de/archive/2007/mobility-biographies-elements-of-abiographical-theory-of-travel-demand (13/02/2019).

"Scheiner, J. (2014). Gendered Key Events in the Life Course: Effects on Changes in Travel Mode Choice Over Time. Journal of Transport Geography, 37, p.47-60.

» Scheiner, J. e Holz-Rau, C. (2012). Changes in Travel Mode use After Residential Relocation: a Contribution to Mobility Biographies. Transportation, 40, p. 431-458.

» Scheiner, J. e Holz-Rau, C. (2013). A Comprehensive Study of Life Course, Cohort, and Period Effects on Changes in Travel Mode Use. Transportation Research Part A, 47, p. 167-181.

» Schoenduwe, R., Mueller, G. M., Peters, A., Lanzendorf, M. (2015). Analysing Mobility Biographies With the Life Course Calendar: a Retrospective Survey Methodology for Longitudinal Data Collection. Journal of Transport Geography, 42, 98-109.

"Seraj, S.; Sidharthan, R.; Bhat, C. R.; Pendyala, R. M. e Goulias, K. G. (2012). Parental Attitudes toward Children Walking and Bicycling to School. Transportation Research Record: Journal of the Transportation Research Board, 2323 (1), p. 46-55. https://doi.org/10.3141/2323-06 
» Simsekoglu, O.; Nordfjaern, T. e Rundmo, T. (2015). The Role of Attitudes, Transport Priorities, and Car Use Habit for Travel Mode Use and Intentions to Use Public Transportation in an Urban Norwegian Public. Transport Policy, 42, p. 113-120.

» Taco, P. W. G. (2003). Redes Neurais Artificiais Aplicadas na Modelagem Individual de Padrões de Viagem Encadeadas a Pé. Tese de Doutorado. Programa de PósGraduação em Engenharia de Transportes, Escola de Engenharia de São Carlos, Universidade de São Paulo. São Carlos, SP.

»Takano, M. S. M. (2010). Análise de Influência da Forma Urbana no Comportamento de Viagens Encadeadas com Base em Padrões de Atividades. Dissertação de Mestrado. Programa de Pós-Graduação em Transportes, Universidade de Brasília. Brasília, DF.

"Takano, M. S. M. (2018). Metodologia para análise dos efeitos dos estágios no ciclo de Vida no Comportamento de Viagem. Tese de Doutorado. Programa de PósGraduação em Transportes, Universidade de Brasília. Brasília, DF.

» Tertoolen, G.; Van Kreveld, D. e Verstraten, B. (1998). Psychological resistance against attempts to reduce private car use. Transportation Research Part A, 32 (3), p. 171-181.

»Van der Waerden, P.; Timmermans, H. e Borgers, A. (2003). The influence of key events and critical incidents on transport mode choice switching behaviour: a descriptive analysis. 1oth International Conference on Travel Behaviour Research, Lucerne, August 2003.

»Zwerts, E.; Janssens, D. e Wets, G. (2010). How the presence of children affects parent's travel behavior. Hasselt University - Campus Diepenbeek, Transportation Research Institute. Disponível em: http://citeseerx.ist.psu.edu/ viewdoc/download?doi=10.1.1.625.156o\&rep=rep1\&type=pdf (31/o1/2019).

\section{Fabiana Serra de Arruda / farruda@unb.br}

Docente do Departamento de Engenharia Civil e Ambiental e do Programa de PósGraduação em transportes da Universidade de Brasília, Brasil. Pesquisadora nas áreas de Mobilidade Urbana, Comportamento Individual de Viagens e Modelagem em Transportes. Currículo Lattes: http://lattes.cnpq.br/2580906230595550

\section{Marcelo Pereira Almeida / marceloalmeidagyn@gmail.com}

Mestre em Transportes e doutorando em Transportes, ambos pelo Programa de PósGraduação em Transportes da Universidade de Brasília. Currículo Lattes: http://lattes. cnpq.br/1037089976151399

\section{Daniel Pires Vieira / vieira.pires@gmail.com}

Docente do Programa de Pós-Graduação em Administração da Universidade de Brasília. Pesquisador nas áreas de Estratégia e Competitividade, Modelos estatísticos aplicados à análise de dados e Políticas Públicas. Curriculo Lattes: http://lattes.cnpq. br/8479518094057050 